\title{
Third issue of volume 12
}

\author{
Matthias F. Carlsohn ${ }^{1} \cdot$ Nasser Kehtarnavaz $^{2}$
}

(c) Springer-Verlag Berlin Heidelberg 2016

As mentioned in previous editorials, this year in 2016 two volumes of JRTIP, volumes 11 and 12, each with four issues are published by Springer Nature. The four issues of volume 11 have already been concluded, and this is the third issue of volume 12 . The additional volume or a total of 1600 additional print pages are devised in order to address the backlog of accepted papers that appear online and are waiting to appear in print. We plan to continue taking steps to steadily shorten the time between the online and print publications of accepted articles.

As normally done in the last few years, the editorial board of JRTIP will be meeting at the SPIE Conference on Realtime Image and Video Processing as part of the SPIE umbrella conference on Defense and Commercial Sensing to be held in April 2017 in Anaheim, CA. The call for papers for the conference can be found here: http://spie.org/SIC/con ferencedetails/real-time-image-video-processing and is included in the back matter of this issue. We will report on the outcome of this meeting in an editorial appearing after the conference.

Before providing a brief outline of the papers in this issue, it is worth mentioning that the newly released ISI impact factor (IF) for JRTIP now stands at 1.564. An analysis of the data used for the IF computation reveals that the drop from the last year's IF of 2.02 is due to the increase in the number of published articles without a

Matthias F. Carlsohn

Matthias.Carlsohn@t-online.de

Nasser Kehtarnavaz

kehtar@utdallas.edu

1 Engineering and Consultancy Dr. Carlsohn for Computer Vision \& Image Communication, Bremen, Germany

2 University of Texas at Dallas, Richardson, TX, USA proportional increase in the number of citations, thus adversely affecting the JRTIP's IF. Note that among 70 journals in Springer Computer Science journals, JRTIP is ranked in the top quarter of these journals and among 255 journals in Engineering, Electrical and Electronics, it is ranked 104. Within the top 20 journals in the category Imaging Science and Photography Technology, JRTIP is ranked 10. The current annual download of 39,000+ of JRTIP articles demonstrates an increasing interest by the real-time image processing community. The fact that more than $2 / 3$ of the downloads are made of the articles published in previous years is a reflection of the usefulness lifetime of the content provided by JRTIP. It is interesting to note that $29 \%$ of the total visits that target the Springer pages indicate JRTIP as the main source for answers to real-time image processing questions.

We also wish to report that the great majority of authors have stated their satisfaction with their publishing experience in JRTIP and have rated their publication experience between excellent and good with $82 \%$ who will consider submitting future manuscripts to JRTIP. For receiving such a highly positive feedback, we wish to thank the authors, reviewers, associate and guest editors and also the publishing team of Springer Nature.

In order to further improve the turnaround time of the review process, we would like to point out the Reviewer Reward Programme whose details we had mentioned in previous editorials and are described at this link: http:// www.springer.com/computer/image+processing/journal/ 11554/PSE?detailsPage=press .

Furthermore, we would like to encourage new proposals for special issues to be offered by guest editors. Such issues provide a collection of focused articles on contemporary subjects of interest as related to the real-time aspects of image processing such as computational complexity 
reduction compared to existing solutions, real-time hardware implementation on various processors or platforms, actual real-time processing rates and real-time software optimization. The calls for papers for special issues that are currently open can be found at the JRTIP's homepage www.springer.com/11554.

Although this third issue of volume 12 appears as a regular issue, it is organized in order to provide a cross section of papers covering a wide span of image processing themes within the context of real-time processing. This issue comprises a total of 7 original research articles addressing different image processing topics.

The first paper by C. Chang et al. entitled "Computationally efficient image deblurring using low rank image approximation and its GPU implementation" presents a GPU implementation of a computationally efficient technique to reduce image blur caused by handshakes in images captured by mobile devices. It is shown that by combining different exposures of images and by applying low rank image approximation, one can achieve a better deblurring performance than competing schemes by examining three different image quality measures.

The second paper by Y. Li et al. entitled "Adaptive mode decision for multiview video coding based on macroblock position constraint model" discusses mode decision, motion estimation and disparity estimation to achieve high compression ratio in multiview video coding (MVC) while reducing computational complexity. The mode decision approach considered uses a macroblock (MB) position constraint model (MPCM) that reduces the number of candidate modes based on mode correlation and rate distortion costs (RD cost) of previously encoded frames/ views. The experimental results show that the proposed method can save approximately $86 \%$ of the encoding time compared with the mode decision used in the reference software of joint multiview video coding, with $0.077 \mathrm{~dB}$ loss in "Bjontegaard delta peak signal-to-noise ratio" (BDPSNR) and $2.3 \%$ increase in the total Bjontegaard delta bit rate (BDBR).

The third paper by S. Yang et al. entitled "SBRISK: speed-up binary robust invariant scalable keypoints" presents keypoint generation, including detection, description and matching as the basis of a broad range of applications to speed-up BRISK (SBRISK), a variant of the binary robust invariant scalable keypoint (BRISK). This solution inherits the high speed of BRISK in the keypoint detection and adopts a nearly circular symmetric constellation to describe the pattern of keypoints. To adapt to the characteristic orientation of keypoints, SBRISK shifts the binary vector rather than rotating the image pattern. The experiments conducted demonstrate that SBRISK is faster and show better performance than BRISK with lower memory consumption.
The fourth paper by E. Smistad et al. entitled "Multigrid gradient vector flow computation on the GPU" presents Gradient Vector Flow (GVF) as a feature-preserving spatial diffusion of image gradients in order to overcome the limited capture range in traditional active contour segmentation. The original iterative solver for GVF, using Euler's method, converges very slowly, i.e., needs a high number of iterations to achieve the desired capture range. The introduced multigrid methods provide a better capture range leading to considerably fewer iterations with the consideration that their CPU-implementations are slower than the Euler's method executed on a GPU. The novelty of this paper is its OpenCL implementation of a multigrid solver for GVF for GPU operation that converges, provides a better capture range, and being 2-5 times faster than the conventional iterative GVF solver running on GPUs.

The fifth paper by A. Maximo et al. entitled "Efficient finite impulse response filters in massively parallel recursive systems" presents strategies to parallelize recursive systems by an algorithm linear in time and memory providing inherent parallelism for GPU implementations. It enables the implementations of closed-form formulas combining non-recursive and recursive linear filters based on an efficient state-of-the-art block-based strategy. As an application of the early stage of vision, an approximation of the Gaussian filters and their first and second derivatives are implemented on a GPU demonstrating real-time image filtering on UHD video signals.

The sixth paper by F. Zhou et al. entitled "Fast star centroid extraction algorithm with sub-pixel accuracy based on FPGA" plays an important role in celestial navigation, providing spacecraft's attitude information that is determined by matching the star's centroid in a captured image with its reference information taken from star catalogs. Star images are often luminance spots of diameters smaller than 5 pixels, making the requirement of sub-pixels accuracy in centroid extraction almost impossible, especially in FPGA-based hardware implementations. Existing spot centroid extraction methods require plenty of pixels to perform the necessary computations with the required accuracy being not suitable for hardware implementation. In the first step, the maximum pixel-level center is located through zero crossing of the first derivative within a small region. In the second step, these pixel-level centers are taken as the middle of a window with fixed size so that subpixel offsets to the sub-pixel center is calculated using a fixed window weighted centroid method. The sub-pixel center of a star is then obtained by adding the offsets to the pixel-level center. A corresponding Verilog design for FPGA implementation is shown to increase the processing speed as demonstrated by simulation results showing high performance in accuracy and processing speed with good noise robustness. 
The seventh or final paper by A.B. Jambek et al. as it is entitled proposes a "Low-energy motion estimation architecture using quadrant-based multi-octagon (QBMO) algorithm." H.264 video coding requires high computational complexity and consumes correspondingly large amounts of energy to process a video sequence, especially during motion estimation (ME) searches. The low-energy ME architecture proposed in this paper overcomes this drawback by utilizing a quadrant-based multi-octagon search algorithm, resulting in fast-search motion estimation and reducing the clock cycle by $42 \%$ leading to a reduction in the corresponding energy consumption by $43 \%$. 